\title{
Emerging Technologies for Driving Road Safety and Traffic Management for Urban Area
}

\author{
${ }^{1}$ Yogesh Golhar and ${ }^{2}$ Manali Kshirsagar \\ ${ }^{1}$ Research Scholar, Department of Information Technology, Yeshwantrao Chavan College of Engineering, \\ Nagpur, Maharashtra, - 441110, India \\ ${ }^{2}$ Department of Computer Technology, Yeshwantrao Chavan College of Engineering, \\ Nagpur, Maharashtra, - 441110, India
}

\section{Article history}

Received: 21-06-2021

Revised: 02-09-2021

Accepted: 28-09-2021

Corresponding Author:

Manali M. Kshirsagar

Department of Computer

Technology, Yeshwantrao

Chavan College of Engineering, Nagpur

Emai: manali_kshirsagar@yahoo.com

\begin{abstract}
The on-road traffic increases due to the exponential growth of the number of vehicles on the road. That leads to undesirable road conditions and makes urban population life uncomfortable. It includes traffic congestion, traffic jams and long queues at traffic signals and toll booths. Many people violate road traffic rules and regulations rules every day. Manual human effort and supervision are not enough to resolve these issues. Still, many road traffic managements follow the traditional approach, which results increase in road accidents that makes humans seriously injured and even loss of life. To address these issues, we have proposed four strategies as (1) congestion status on square, (2) efficient toll tax collection, (3) fine collection on road for the people who violate road traffic rules, (4) shortest pathfinder for drivers. This research work mainly focuses on the various strategies and their implementation with the help of the Map-Reduce framework. We have combined video surveillance, big data analytics algorithm and proposed an innovative way to manage and handle the road traffic.
\end{abstract}

Keywords: Map Reduce, Road Traffic Management, Big Data Analytics, Hadoop Framework

\section{Introduction}

In India travel by highway is risky because of two reasons (1) due to road conditions, (2) Irresponsible behavior of drivers on road leads to accidents. India is at the top position in the world in traffic-related deaths. In other countries like U.S. people suffered from severe traffic accidents in recent times. Every person should be more attentive while walking side by road, specifically in the area of pedestrian walking zones. Every four and six-wheeler driver must use safety seat belts. Each two-wheeler driver must use the Helmets. Travel in the evening is especially dangerous. Buses, habituated by hundreds of millions of Indian people. It is observed by the traffic authority many drivers are driving fast and fearlessly in the congested areas. Results in on road accidents are quite obvious. However, robust traffic monitoring and management system is required to deal with such problems in India and other countries. Current traffic management system is manual. Human traffic controller monitors and manage the on-road traffic in India. At, many places the surveillance camera are used to monitor the traffic and capture traffic rules and regulation scenarios. But not all the roads, are under surveillance in India. Existing on road traffic surveillance cameras-based traffic management system have different issues and challenges such as huge traffic record management, active surveillance missing on road, because in current system no automated fine collection module is not included. It is getting enhance day by day in India. Recently, Traffic control management organization in India comes with the initiative of FastTrack system on toll booth. It is used for the collection of tax on toll booth for avoiding the long queue on the road. In FastTrack system, every vehicle has their own unique QR code attached with the account.

Whenever, the vehicle comes on toll both, the camera on the toll booth mounted on the top scanned the QR code of vehicle. Then amount is automatically deducted from the account without any human involvement. But, issue with FastTrack system include, (1) vehicle and camera mounted camera calibration, position of vehicle should be appropriate to capture the QR code on vehicle. (2) Quality of camera on toll booth is poor. Sometimes it is difficult for vehicle driver to positioned the vehicle on the toll booth. We try to address these issues with our proposed strategies of traffic management using big data analytics. 
In proposed work we have come with four efficient strategies to overcome the existing system drawbacks. The proposed framework utilized the Map Reduce framework to manage the traffic big data efficiently. The four strategies include (1) traffic status on the road based on the congestion degree, (2) efficient toll tax collection, (3) fine collection on road for the people who violate road traffic rules, (4) shortest path finder for drivers. The proposed approach will resolve the aforementioned issues and challenges of traffic monitoring and management system.

\section{Literature Survey}

Nowadays, ruler people are moving towards the urban cities due to better job opportunity and urban road traffic increase day by day. In the upcoming years approximately $70 \%$ of the world population expected to live in urban cities (Dobbs et al., 2011). The government of India Smart Cities announced the fund of 160 billion for making the 100 smart cities of country (Abbu et al., 2015; Nandury and Begum, 2015).

Javaid et al. (2018), proposed distributed and centralize approach to manage road traffic. The traffic information collected using the video surveillance camera and automated signal changes automatically. Cloud platform used as a storage to store and manage large size videos. Hence, there is a risk of single source failure. Swathi et al. (2016), introduced an efficient traffic routing system, less congested road path was suggested to the end user. Different infrared and image sensors are utilized to collect the road traffic. However, change in illumination, temperature variation, overcast condition and humidity affects the traffic density values which is based on the weather situation. Wiering et al. (2004), proposed adaptive control system to control traffic light of roads. In this system based on on-road traffic condition traffic light color changes adaptively. Due to this long queue on signal get reduce, the drivers can always take the lowest estimated waiting path for reaching destination. In this system, if all the drivers select or opts for the same route, the optimal and less time-consuming path becomes overcrowded and thus, the system may become inefficient for all.

Calvert et al. (2018), proposed improved traffic management system by taking into consideration of the uncertain and unpredictable behavior of traffic flow. Kim et al. (2018) proposed a remote location one fingertip mobile application and cloud-based traffic management system. The system uses the intelligent traffic monitoring and navigation system using formal methods and graph theory techniques. This system works efficiently when the on-road traffic is heavy, this system will be more effective as manual traffic management system does not work well in emergency situations. Further, the drivers notified with the alternative path notification.

Latif et al. (2018), introduced smart road transportation approach to efficiently control the heavy congestion areas on the road and managing the on-road accident. Liu et al. (2013), introduced the intelligent and active traffic monitoring system using a RFID sensor, it creates a custom routing map of the shortest and less time-consuming map of the city. It will help drivers and traffic authorities to get overviews of traffic of whole city.

Currently, traffic data collected using video cameras, sensors, etc. Video camera used for video analysis, in this the smart cameras with high quality image sensors, processing and communication entities. These cameras are used to monitor the traffic. Traffic information statists are computed using the scene description and video understanding. These include vehicle type, frequency, number plate detection, average speed of vehicles and lane occupancy information, etc. Liu et al. (2013). The onroad sensors sometimes called as Traffic detectors (Manikonda et al., 2011). The application includes such as network traffic measurement, traffic monitoring and control, computation of speed of vehicle, on road accident detection.

Infrared embedded sensors are divided into two categories as (1) passive detector and (2) active detectors. Passive type extracts the information from environment specifically it records the information reflected or generated from the object. It can be useful to compute the speed, length, number of vehicles and dimension of vehicle. Active type propagates the signal and received the reflected energy from the object. Ultrasonic object detectors (Manikonda et al., 2011) transmit the wave in omni direction and gather the information available in the environment. Hadoop Distributed File System (HDFS) is utilized for storing the big data. The master and slave architecture adopted by the HDFS. It exposes a file system namespace and allows user data to be stored in files. HDFS is used for processing the Big Data efficiently.

Recent literature survey uses different technologies and strategies for efficient traffic management framework. Cárdenas-Benítez et al. (2016) proposed traffic congestion detection using connected component analysis. It includes two approaches (1) traffic event detection algorithm and (2) route Monitoring Algorithm useful for road incident detection and navigation. The algorithms utilized the simulated data generated using SUMO (Simulation of Urban Mobility) traffic simulator. This approach is not suitable for real-time road traffic scenarios and situation aware routing is not possible using this model. Gupta et al. (2013) proposed a Detect Traffic Congestion (DTC) by Mining versatile GPS data model. It includes (1) identification of On-Road Clusters Algorithm, (2) Binary Traffic Output Algorithm. The source of data for these approaches are GPS enabled devices like mobiles, tablets and from vehicles etc. The limitation of this is misclassification of traffic jam scenarios due to in-accurate vehicle of GPS location. Ganesh and Appavu (2015) an Intelligent Video System (IVS) Framework with Big Data Management for Indian 
Road. The limitation of this approach includes (1) not suitable for large scale deployment, (2) misclassification of traffic jam scenarios due to in-accurate vehicle localization. Adoni et al. (2017) proposed a traffic event detection using Map Reduce framework. The input traffic event $\log$ s are generated using the Apache flume. The limitation of this framework includes (1) time complexity for traffic event detection is more, (2) advanced traffic analysis algorithm can be integrated to this framework for improve the processing of traffic event logs, (3) reuse the same approach for traffic congestion detection can enhance the performance this framework. Due to existing system limitation in real time environment, it is observed that there is need of efficient traffic management system to handle the current traffic scenarios in the urban environment.

\section{Proposed Methodology}

The proposed approach is classified into three stages as (1) Road traffic information captured using video camera, (2) Extraction of traffic information from video frames, (3) Map Reduce framework for predicting traffic states. Again, proposed work further classified as, A. video processing/analysis i.e., preprocessing of video footages. B. storage of data in HDFS and analyzing it using Hadoop to find the congestion degree. Pre requisite for this project: Implementing camera for recording traffic information on various prominent location. This will detect the types of vehicles, vehicles speed, then calculating a congestion degree. Implement a complete system for Vehicle Registration system with various other modules, such vehicle registration, fine collection system, toll tax collection, shortest path.

\section{A. Video Analysis}

The vehicle in a video detected and classified using the vehicle detection and classification algorithm. It uses HAAR cascade classifier De Souza et al. (2017). It describes in brief as follows.

\begin{tabular}{c}
\hline Algorithm-1: Vehicle detection and classification \\
Step 1: a. Video $\rightarrow$ Converted to frames (25f/s) \\
b. Tot Count $\rightarrow 0$
\end{tabular}

Step 2: By using HAAR cascade algorithm following information is extracted

$\int(x, y, w, h)$

Step 3: Moving objected represented by rectangular box defined by: $\left(x_{1}, y_{1}\right),\left(x_{2}, y_{2}\right)$ where $x_{2}=x_{1}+w, y_{2}=y_{1}+h$ Step 4: Finding the centroid $C=\left(x_{1}+\left(\frac{w}{2}\right)\right),\left(y_{1}+\left(\frac{h}{2}\right)\right)$

Step 5: Input the training set for cascade classifier

Step 6: Frame by frame reading of video

Step 7: Detection of ROI of lane represented as spatial (x, y) coordinate location.
Step 8: if length $($ rectBox $) \neq 0$ then

Total vehicle $=$ Total vehicle +1

If area of the rectangle is less than 10,000 classified it as bike $\{$ Increment bike value by 1$\}$

If area of the rectangle is 10,000 to 17,000 classified it as car $\{$ Increment car value by 1$\}$

If area of the rectangle is greater than 17,000 classified it as truck/bus $\{$ Increment bus value by 1$\}$

Step 9: For each video frame repeat step two to step eight otherwise End.

Step 10: End

(where,

$x, y=$ are the coordinated,

$w=$ width,

$h=$ height,)

The Table 1 shows the types of vehicles and classification category of vehicle utilized in the proposed algorithm:

The Table 2 shows the vehicle classification category based on the speed utilized in the proposed algorithm:

The above data and classification useful in extracting the information such as, number of vehicles on road, road speed, etc.

\section{B. Storage of data in HDFS and Congestion degree computation using Hadoop}

The extracted parameter in algorithms is used by Mapper Reducer function in HDFS for processing data in Hadoop. The congestion degree is computed to get the current status of traffic state using following algorithm.

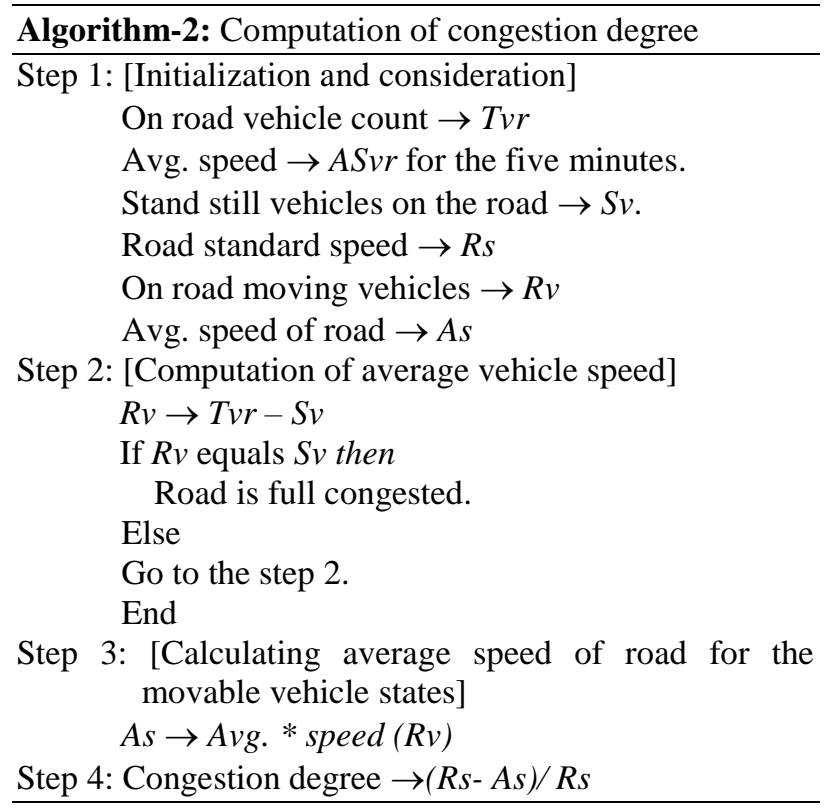

Table 3 shows the computed congestion degree will vary from 0 to 1 as per the traffic congestion and it is divided into different category as follows: 
Table 1: Classification of vehicle based on wheels

\begin{tabular}{ll}
\hline Vehicles types & Vehicles category \\
\hline 2 wheelers vehicle & Scooter, Bike, Scotty, etc. \\
3 wheelers vehicle & E-Riksha, Auto, Micro \\
& transportation vehicle, etc. \\
4 wheelers vehicle & LMV, Car, \\
More than 4 wheelers vehicle & Heavy Trucks, Bus \\
\hline
\end{tabular}

Table 2: Classification of vehicle on the speed parameter

\begin{tabular}{ll}
\hline Vehicle Speed & Vehicles speed classification \\
\hline $0.0 \mathrm{Km} / \mathrm{h}$ & Vehicle stationary \\
$0-25 \mathrm{Km} / \mathrm{h}$ & Vehicle moving slow \\
$26.0-50.0 \mathrm{Km} / \mathrm{h}$ & Vehicle moving normal \\
$51.0-75.0 \mathrm{Km} / \mathrm{h}$ & Vehicle fast moving \\
Above $75.0 \mathrm{Km} / \mathrm{h}$ & Vehicle moving very fast \\
\hline
\end{tabular}

Table 3: Congestion degree distribution

\begin{tabular}{ll}
\hline Congestion degree & Traffic category \\
\hline 0 to 0.25 & Heavy congestion or stand still \\
0.26 to 0.50 & Congestion \\
.50 to 0.75 & Slow moving \\
0.75 to 1 & No congestion \\
\hline
\end{tabular}

For every $5 \mathrm{~min}$ the congestion degree is computed and also stored and processed in HDFS. Each hour having 5 traffic states and 288 traffic states for $24 \mathrm{~h}$. Again, prior historical information is considered to compute the peak traffic hours as illustrated follows. Table 4 illustrate the categorization of range of congestion degree, traffic type, peak traffic time in the $24 \mathrm{~h}$.

The decision tree used for the detection of congested region, time of peak traffic on the road based on the congestion degree. The Mapper Reducer function implemented on the HDFS to process the traffic log information to predict the traffic states. Next, we have detected the number plate of vehicle and obtain the vehicle unique vehicle number extracted from the number plate.

\section{Algorithm-3: License Plate Identification (LPI) and detection}

Step 1: Read input video from Database.

Step 2: Convert into frames and write each frame in the output directory.

Step 3: Convert input frame into gray scale image

Step 4: Generate binary image i.e., Black and white image from gray scale image.

Step 5: Find shape of image. [It includes resolution of image i.e., dimensions matrix]

Step 6: Generate the label image. In this step connected regions in a binary image are identified and group together.

Step 7: Initialize the matrix for number plate detection (maximum width, height and minimum width and height that a license plate).

Step 8: Find the regional properties using region props. It creates a list of properties of all the labelled regions.

Step 9: Consider the area property. If the region area is so less than it is not considered as number plate.

Step 10: Ensuring that the region identified satisfies the condition of a typical license plate initialize in the step 7 .
Step 11: Add bounding box coordinate on the binary image. Step 12: Drawing rectangle red border color box around number plate.

Step 13: End

The detected number plate used as input the character segmentation algorithm to detect the character in the number plate. Each of step is describe as follows.

Algorithm-4: Character segmentation from detected number plate.

Step 1: Detected number plate is given as input then; Invert was done so as to convert the black pixel to white pixel and vice versa

Step 2: Plot the bounding box on the gray scale image to show the box on the gray scale image.

Step 3: Initialize the character dimension bounding box width and height. It is needed to identify the characters in the number plate independently. [The next two lines is based on the assumptions that the width of a license plate should be between 5 and $15 \%$ of the license plate and height should be between 35 and $60 \%$ this will eliminate some].

Step 4: The character and digit in a number plate is represented in a box with a dimension $20 \times 20$

Step 5: Draw Red border rectangle around the digits

Step 6: Resize the characters to $20 \times 20$ and then append each character into the characters list.

Step 7: Plot the bounding boxes around each character Step 8: Initialized the model which is pre-trained using multiple character combination define in the train directory Step 9: Pass each identified character to the train model to identify each character uniquely from the number plate Step 10: The identified characters are generated from the trained model after testing.

Step 11: End

The extracted traffic information from the input source it is process in HDFS. Figure 1 shows the MapReduce framework of the proposed approach. First, the input data is uploaded in the HDFS in the forms of clusters, later the data is divided into key value pair at different nodes using Mapper function. Then it is shuffle in next stage before reducer process the input data. At the end, the Reducer function group together the homogeneous data into different categories with respective to the key value pair. The consolidate result obtain at the end without any ambiguity.

The proposed system uses the Hadoop environment to processed the input data. The processed output result in traffic jam or no traffic jam as shown in Fig. 2. The input source information include speed, vehicle category, congestion degree, road average speed, congestion status, geo location of vehicle, etc. the Mapper Reducer function design in such way that it will process all of these parameters at once in Hadoop distributed environment. Figure 3 shows the Mapper Reducer function utilized for the different traffic log information. 
Table 4: On traffic type based on the video footage of $24 \mathrm{~h}$

\begin{tabular}{lll}
\hline Range of Congestion Degree & Traffic type & Time slot in $24 \mathrm{~h}$ format \\
\hline $0.0-0.25$ & No congestion on road & Slot: 0 to 8 and 14 to 16 and 23 to 24 \\
$0.26-0.50$ & Slow moving traffic & Slot: $9,12,17,22$ \\
$0.50-0.75$ & Congestion state & Slot: $10,13,21$ \\
$0.75-1.0$ & Heavy congestion on road & Slot: 11,18 to 20 \\
\hline
\end{tabular}

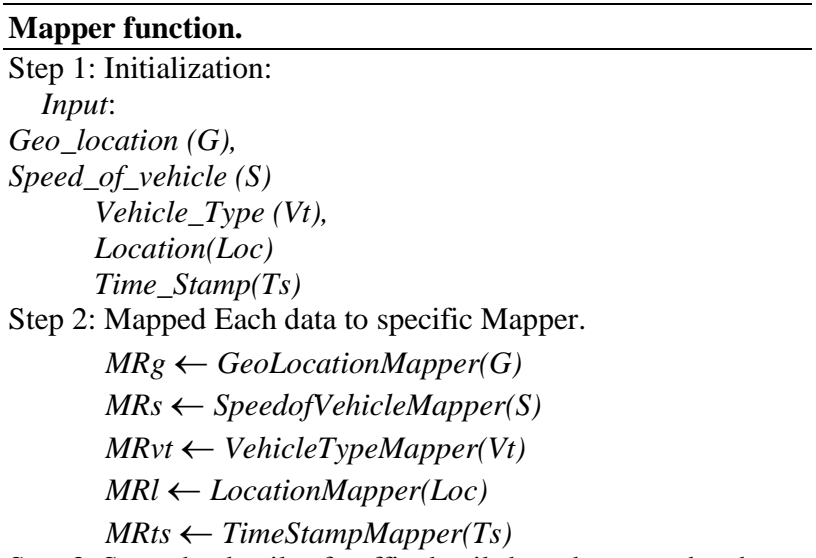

Step 3: Store the details of traffic details based on speed and type in HDFS for road ID.

CongesDegree $\leftarrow$ ComputeCongestionDegree $(S, R D)$

Reducer function.

Step 1: Initialization:

Input: Mapped Input

$$
\begin{aligned}
& M R g \leftarrow \text { GeoLocationMapper }(G) \\
& M R s \leftarrow \text { SpeedofVehicleMapper }(S) \\
& M R v t \leftarrow \text { VehicleTypeMapper }(V t) \\
& M R l \leftarrow \text { LocationMapper }(\text { Loc }) \\
& M R t s \leftarrow \text { TimeStampMapper }(T s)
\end{aligned}
$$

Step 2: Each reducer will generate the output.

$$
\begin{aligned}
& R g \leftarrow \text { ReducerGeoLocation }(\text { MRg }) \\
& \quad R s \leftarrow \text { Reducer Speed of Vehicle }(M R S) \\
& R v t \leftarrow \text { ReducerVehicleType }(M R v t) \\
& R l \leftarrow \text { ReducerLocation }(M R l) \\
& \text { Rts } \leftarrow \text { ReducerTimeStamp(MRts) }
\end{aligned}
$$

Step 3: Store the details of reducer output in HDFS for road ID.

The above Mapper Reducer function is used for the traffic state detection at the end. Later all of the processed data visualized on the Map using geolocation. The toll tax collection and fine collection mechanism is implemented on cloud platform, in which detected number plate and other driver related information store in database and all the status of collected fine or toll tax visualize on the map using geo location. The shortest path routing navigation feature also embedded in the framework for finding the shortest routing path between different location. In the next section we have describe the all the experimented conducted.

\section{Experimental Results}

The road traffic information gathers from various region of Nagpur city. The Fig. 4 illustrate the camera setup on the bridge to record the video of different road lane at different angles $5^{\circ}, 20^{\circ}$ and $25^{\circ}$ from the ground position. The camera place $25 \mathrm{~m}$ away from the road specifically on the bridge. The position camera allows to record the on-road vehicle information in both direction as show in the Fig. 4. The blue and read dash lines shows the cameras range.

The daily road traffic capture using DSLR camera shown in Fig. 5. The left side image shows the raw video frame recorded by the camera placed at the top of the bridge. Right side image consists of ROI marked with white color in rectangle box shape to represent the region from which vehicle information is calculated. The different information extracted from the video such as vehicle speed, vehicle type, number of vehicles in the lane. The two vertical solid lines on the frame shows the road specific region.

Next, the traffic information such as speed of vehicle identified from Region of Interest (ROI). The traffic information proposed using HDFS using Mapper Reducer batch processing unit. On road traffic information useful for computing the congestion degree to predict the traffic state of road. The processed result in HDFS shown in Fig. 6. (a) Directory structure of HDFS where physically computation of files is performed. (b) Job processed output in HDFS using double Map Reduce framework. (c) Computation of type of vehicle using vehicle processing algorithm and (d) Computation of Speed of vehicle using vehicle speed computation algorithm. (e) Congestion degree computation for traffic data.

The license plate Identification system is developed in the Phyton. The hardware configuration used for the implementation of the algorithm is Intel Core i3 CPU@ 3.00 GHz, 16.00 GB RAM, 64-bit operating system. The Video camera used for capturing the video frames is DLSR camera with progressive scan and CMOS sensor. 1280X1024, 25/30fps, H.264/MJPEG/MPEG4, Triple stream, 30 m IR. The camera is manually recording the incoming and outgoing vehicles on the various location of the road. The output of license plate identification system Fig. 7.

The four strategies user interaction shown in Fig. 8. Figure 8(a) shows the congestion status using congestion degree geolocation wise shown on the map. Figure 8(b) shows the toll tax status and collected amount on screen. The map implemented are dynamic in which if end user clicks on the desired toll tac location point. The alert will show the toll tax collection status on screen. Similarly for the fine collection. At last shortest pathfinder for drivers allows end user to select the source and destination location and suggest the shortest path between the selected location. 
The database consists of all the traffic log and event details. Table 5 shows the extracted information from input video. It also divided into different location on road, other data traffic scenarios, size of information with its duration and attribute extracted from the algorithms after processing of source raw data.

The proposed approach compares with the existing approach based on the time complexity. It is observed that the proposed approach requires less time compare to the approach of (Cárdenas-Benítez et al., 2016; Gupta et al., 2013; Ganesh and Appavu, 2015; Adoni et al., 2017). The four strategies are useful for efficient traffic management. The result comparison of proposed system with the existing approaches are shown in Table 6.

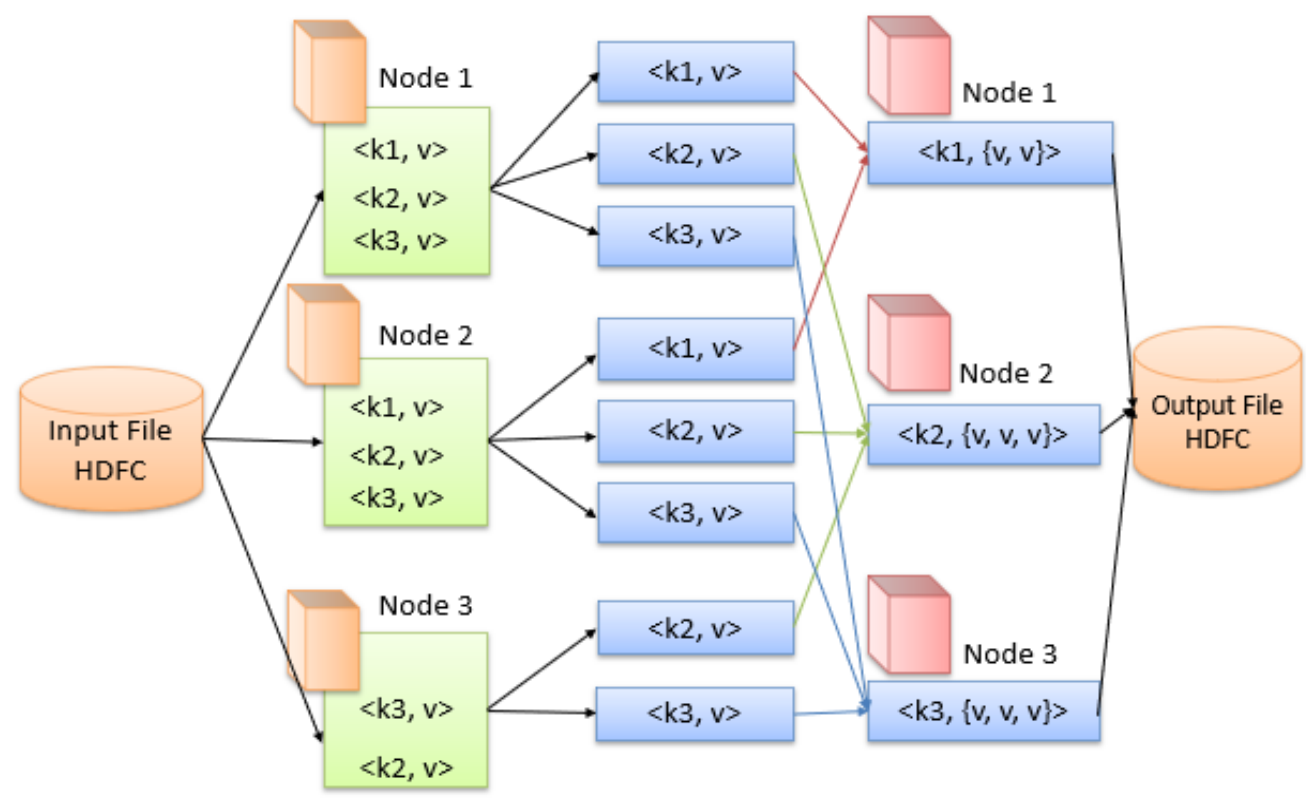

Fig. 1: Map Reduce framework of proposed system

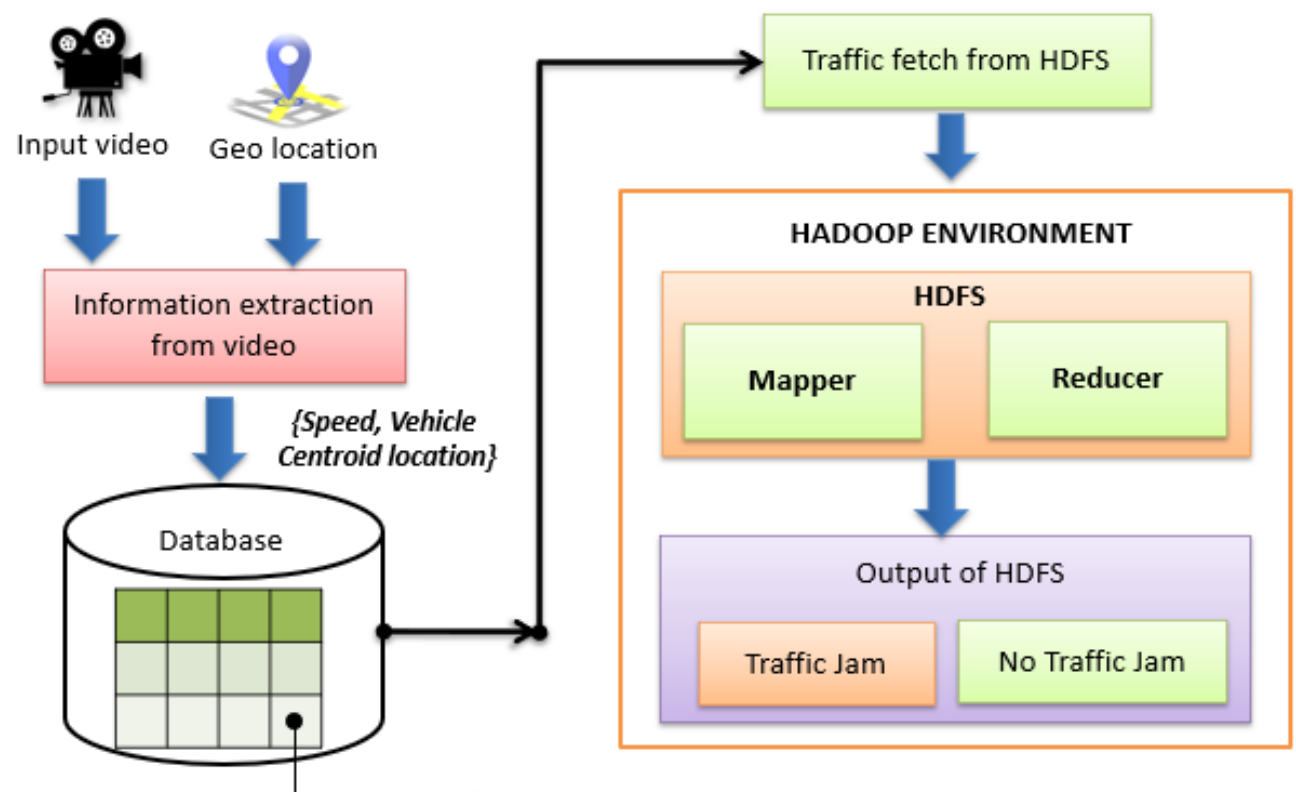

\{Speed, Vehicle Centroid location, Geo location, Road ID\}

Fig. 2: Proposed system architecture 
Yogesh Golhar and Manali Kshirsagar / Journal of Computer Science 2021, 17 (11): 1104.1115 DOI: $10.3844 /$ jessp.2021.1104.1115

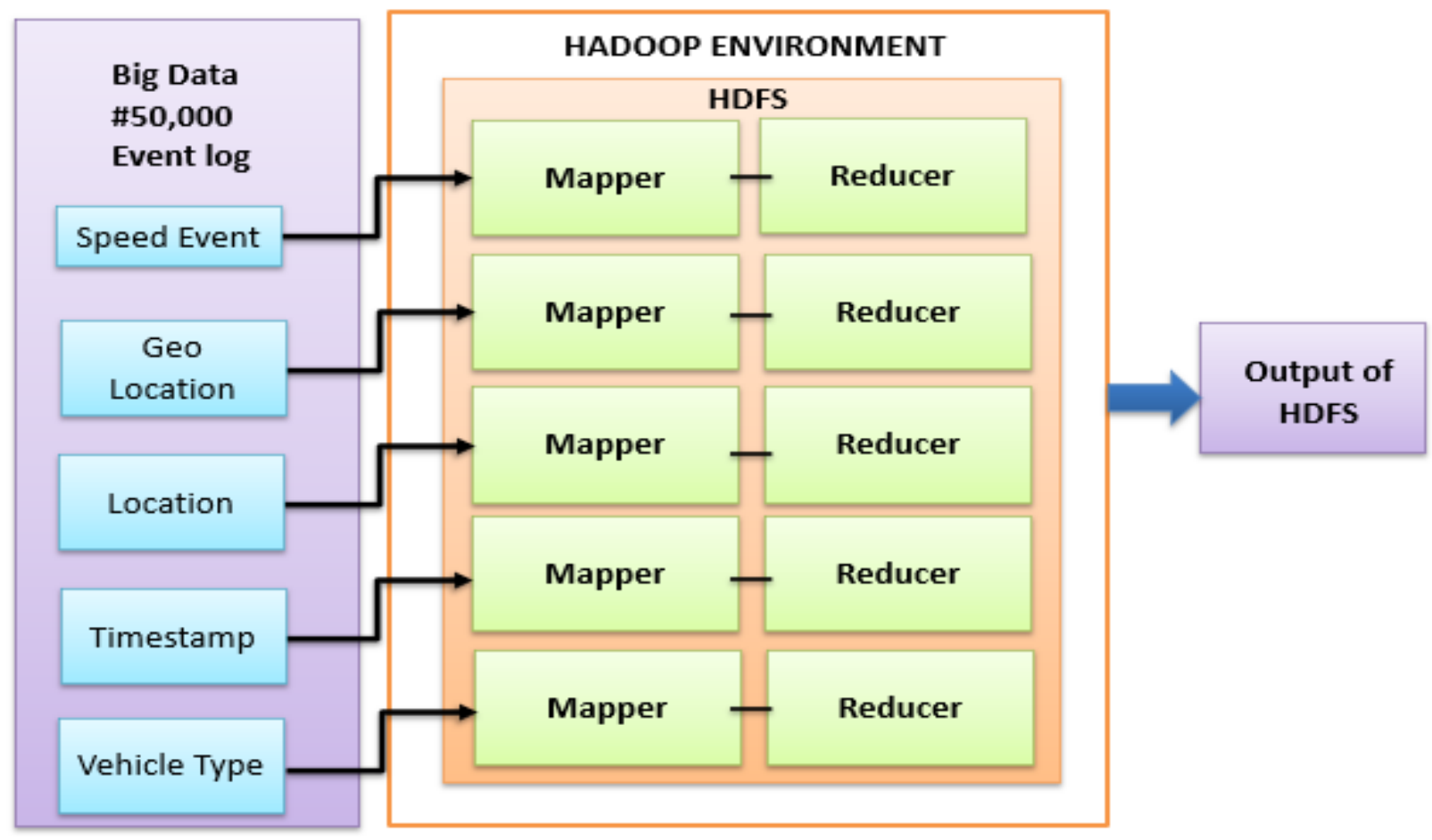

Fig. 3: HDFS input data process using Map Reduce framework

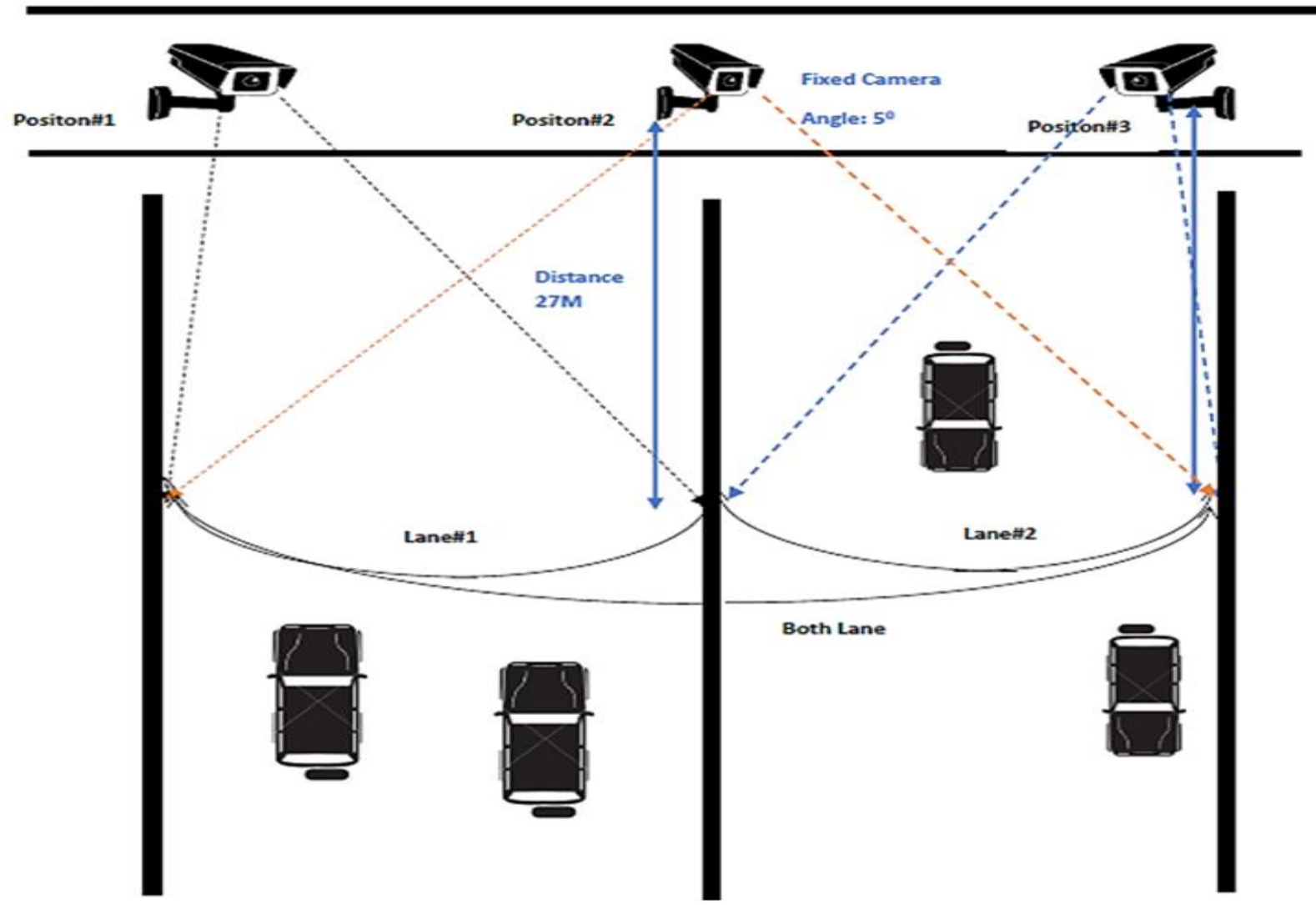

Fig. 4: Experimental setup to record on road highway traffic from bridge 

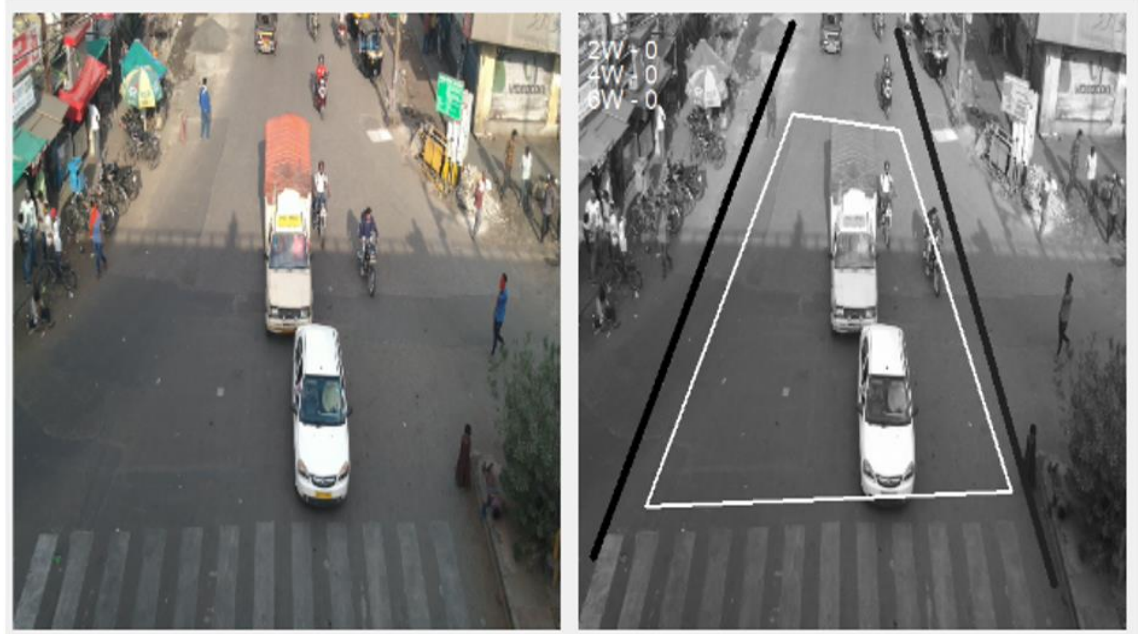

Fig. 5: On road traffic video footage. (a) Top view of road (frame of video) (b) Camera cali-brated video frame
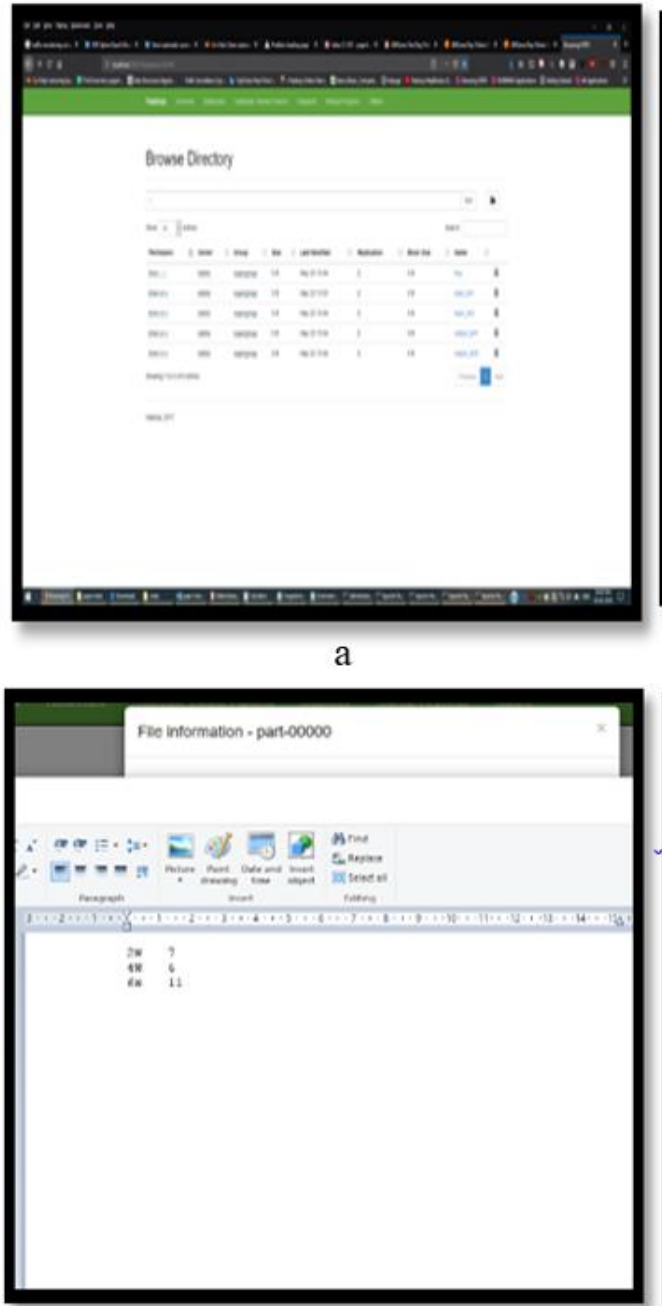

(c)

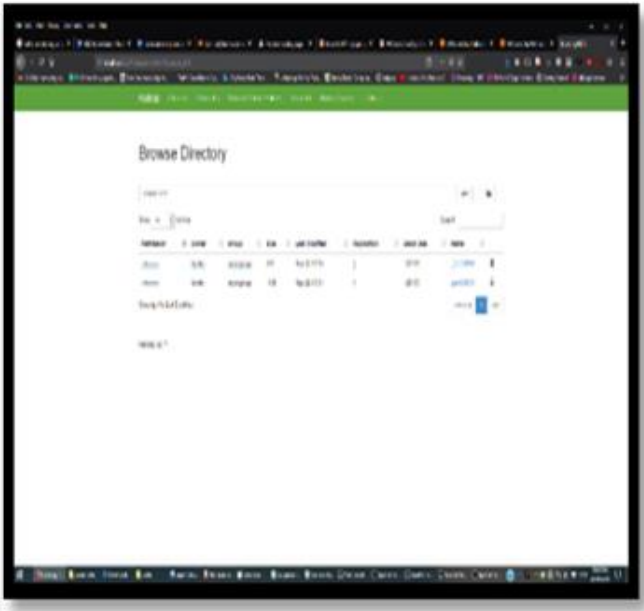

b

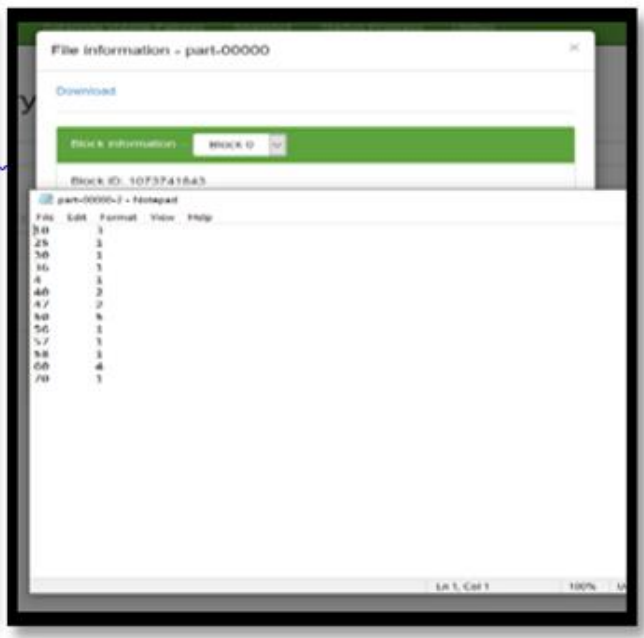

(d)

Fig. 6: (a) Directory structure of HDFS. (b) Job processed output in HDFS. (c) Computation of type of vehicle and (d) Computation of Speed of vehicle 
Yogesh Golhar and Manali Kshirsagar / Journal of Computer Science 2021, 17 (11): 1104.1115

Image Analysis
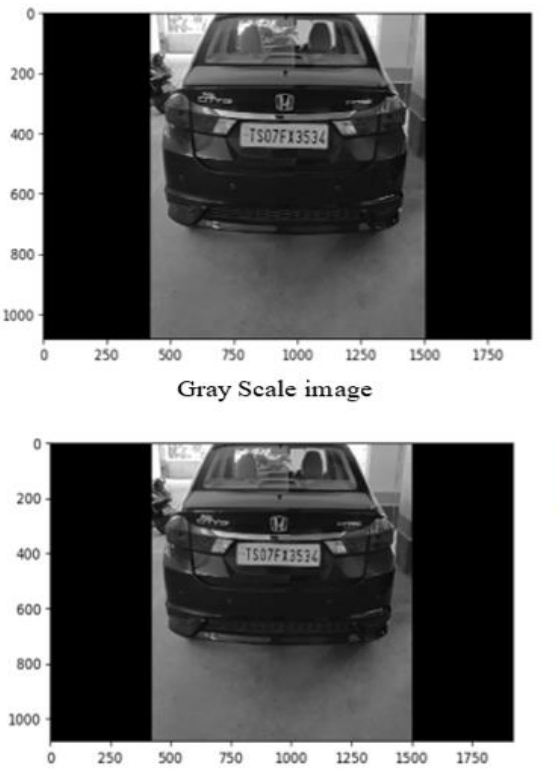

Number Plate character recognition
Black and white image

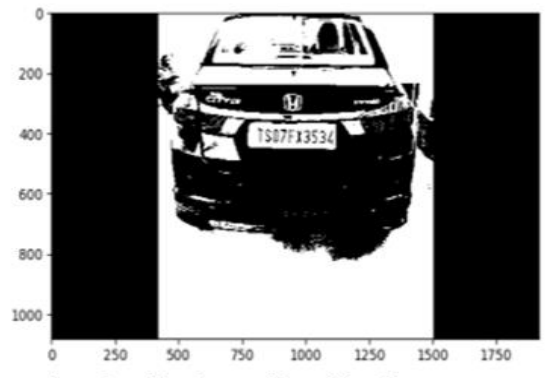

Number Plate block area identification

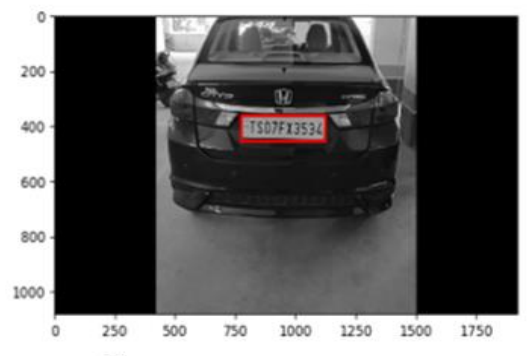

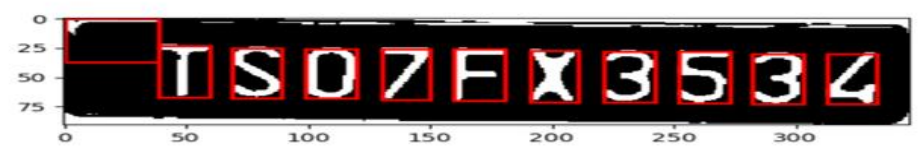

Fig. 7: Number plate detection and character classification

Strategies for Smart Road Traffic Management System

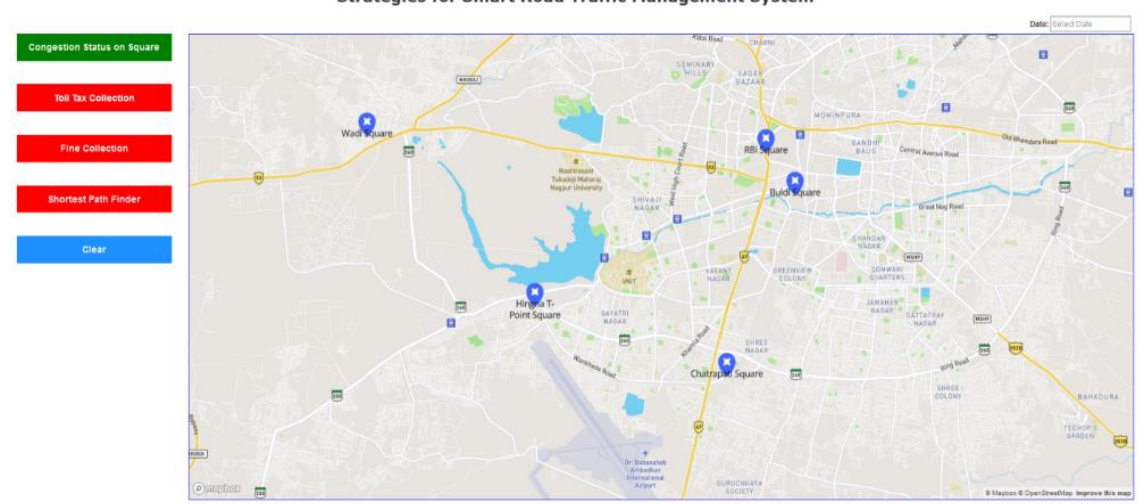

(a)

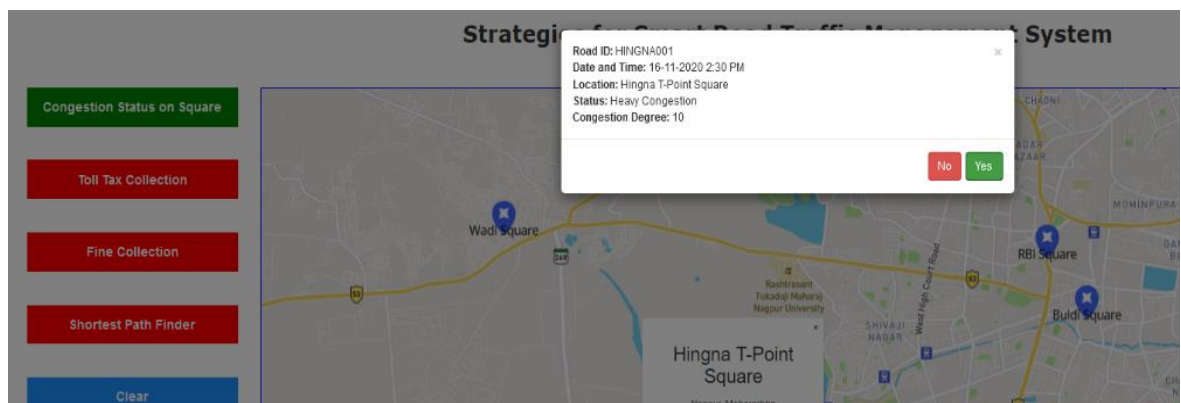

(b) 


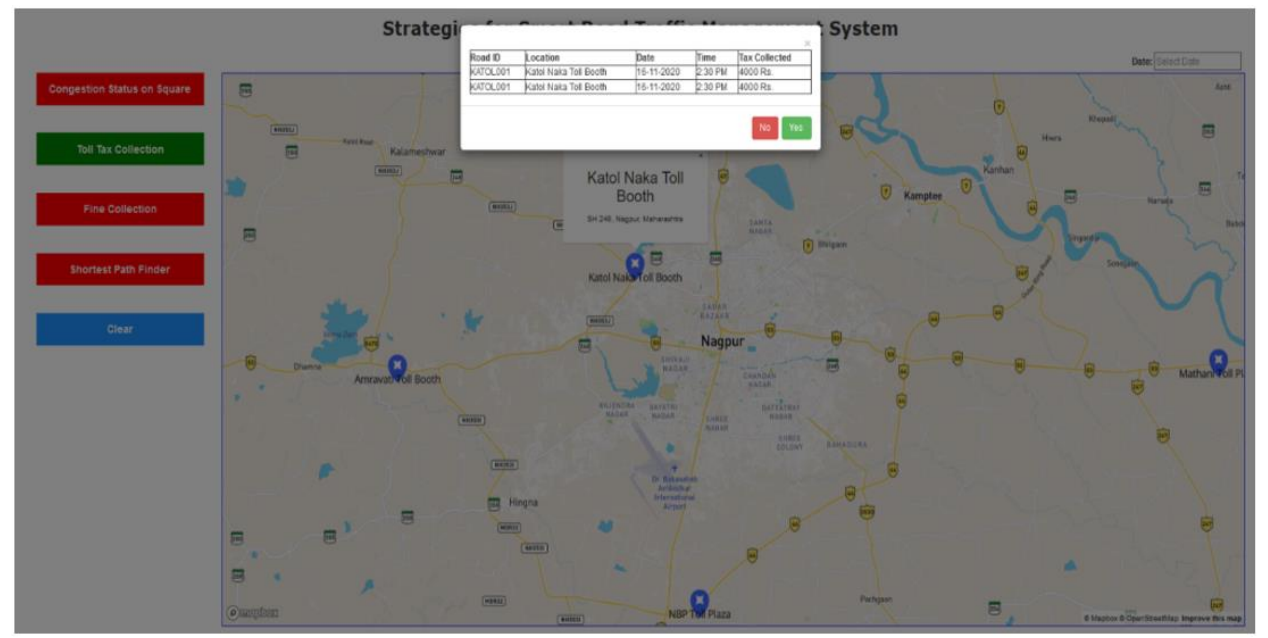

(c)

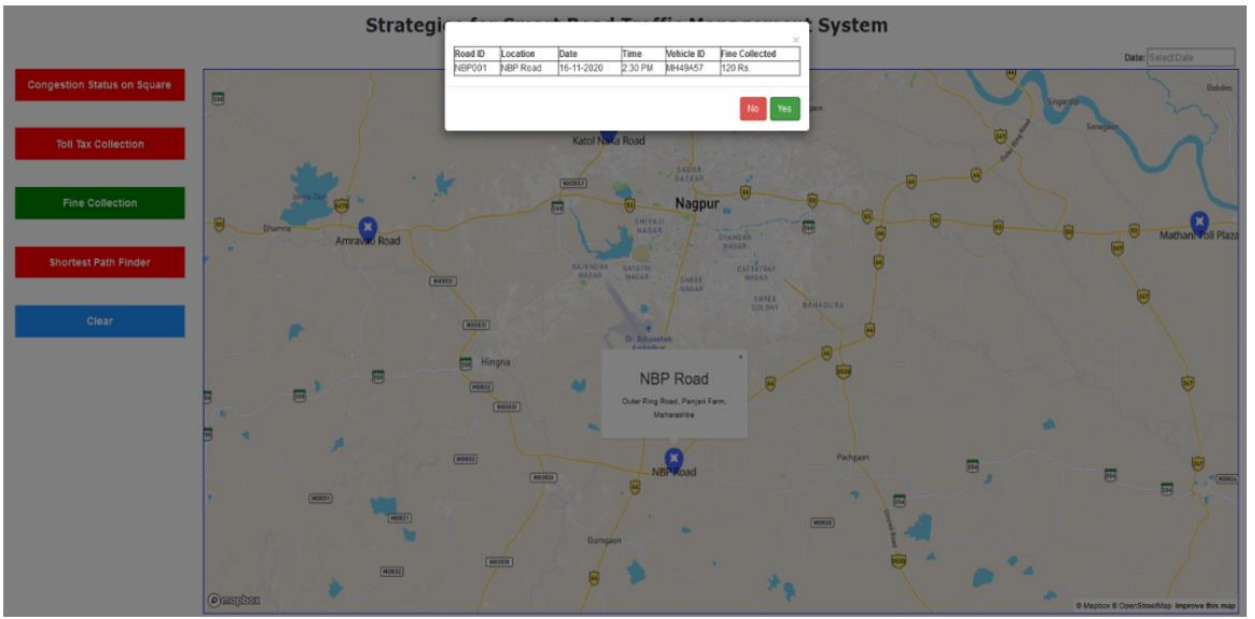

(d)

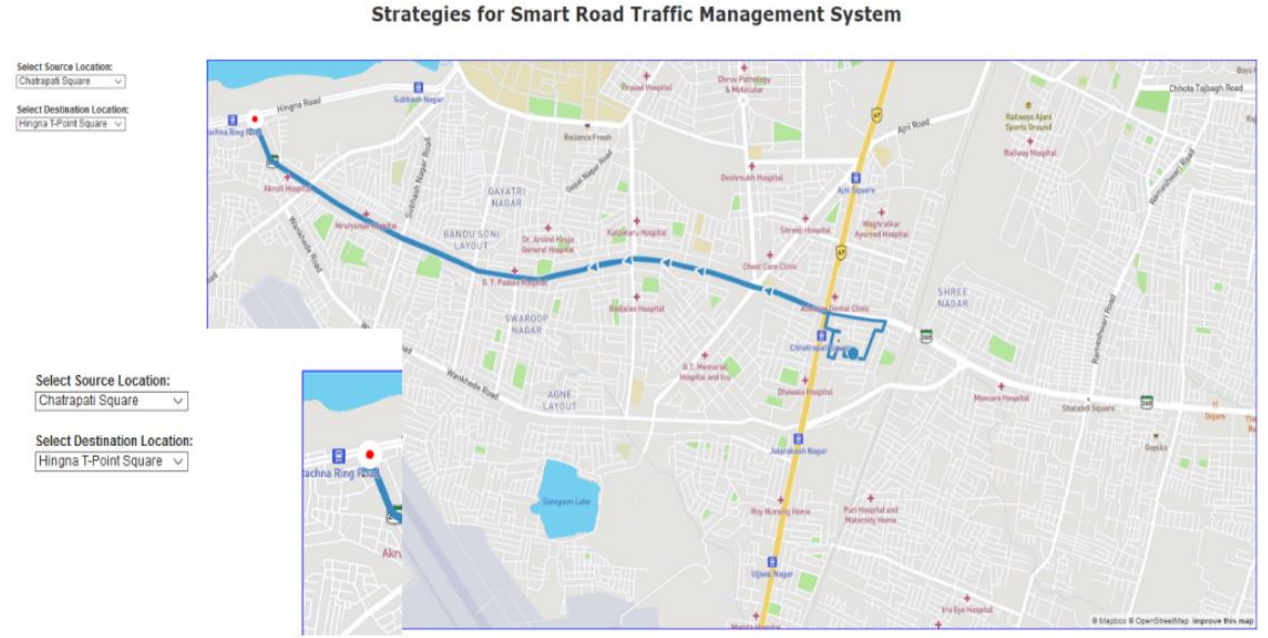

(e)

Fig. 8: (a) Dashboard of Traffic Management System. (b) Congestion status using congestion degree. (c) Toll booth toll tax collection status. (d) Fine collection system on square and (e) Shortest path navigator for drivers 
Table 5: Database information of proposed architecture

\begin{tabular}{|c|c|c|c|c|c|}
\hline \multicolumn{2}{|c|}{ Sr. No. Location } & \multirow{2}{*}{$\begin{array}{l}\text { Traffic Scenario } \\
\text { On Road }\end{array}$} & \multirow{2}{*}{$\begin{array}{l}\text { \#Samples } \\
10 \mathrm{k} \text { videos }(30 \mathrm{f} / \mathrm{s})\end{array}$} & \multirow{2}{*}{$\begin{array}{l}\text { \#Duration } \\
6 \mathrm{hr}\end{array}$} & \multirow{2}{*}{$\begin{array}{l}\text { \#Attributes } \\
\text { Traffic information extracted } \\
\text { from videos is as follows: }\end{array}$} \\
\hline 1 & Buldi Square, Nagpur & & & & \\
\hline 1. & Vehicle Speed, & & & & \\
\hline 2. & Vehicle category, & & & & \\
\hline 3. & Congestion status, & & & & \\
\hline 4. & Congestion type, & & & & \\
\hline 5. & Traffic state for each frame & & & & \\
\hline \multirow[t]{2}{*}{6.} & Geospatial location, time stamp log. & & & & \\
\hline & & Other data & 10k traffic event logs & & \\
\hline \multirow[t]{2}{*}{2} & RBI Square, Nagpur & On Road & $10 \mathrm{k}$ videos $(30 \mathrm{f} / \mathrm{s})$ & $6 \mathrm{hr}$ & \\
\hline & & Other data & 10k traffic event logs & & \\
\hline \multirow[t]{2}{*}{3} & Mihan Square, Nagpur & On Road & $10 \mathrm{k}$ videos $(30 \mathrm{f} / \mathrm{s})$ & $6 \mathrm{hr}$ & \\
\hline & & Other data & $10 \mathrm{k}$ traffic event logs & & \\
\hline \multirow[t]{2}{*}{4} & Chatrapati Square, Nagpur & On Road & $10 \mathrm{k}$ videos $(30 \mathrm{f} / \mathrm{s})$ & $6 \mathrm{hr}$ & \\
\hline & & Other data & $10 \mathrm{k}$ traffic event logs & & \\
\hline \multirow[t]{2}{*}{5} & Hingna T-point, Nagpur & On Road & $10 \mathrm{k}$ videos $(30 \mathrm{f} / \mathrm{s})$ & $6 \mathrm{hr}$ & \\
\hline & & Other data & $10 \mathrm{k}$ traffic event logs & & \\
\hline \multirow[t]{2}{*}{6} & Wadi Square, Nagpur & On Road & $10 \mathrm{k}$ videos $(30 \mathrm{f} / \mathrm{s})$ & $6 \mathrm{hr}$ & \\
\hline & & Other data & $10 \mathrm{k}$ traffic event logs & & \\
\hline
\end{tabular}

Table 6: Comparative analysis of proposed traffic management system with the state-of-the art approaches.

\begin{tabular}{|c|c|c|c|c|}
\hline Sr. No. & Name of author & Algorithms/Methodology & Dataset & Time complexity \\
\hline$\overline{1}$ & Cárdenas-Benítez et al. (2016) & $\begin{array}{l}\text { 1. Traffic event } \\
\text { detection algorithm, }\end{array}$ & SUMO event logs & $20 \mathrm{sec}$ \\
\hline 2 & Gupta et al. (2013) & $\begin{array}{l}\text { 2. Route monitoring algorithm. } \\
\text { 1. Identification of on- } \\
\text { road clusters algorithm } \\
\text { 2. Binary traffic output algorithm }\end{array}$ & GPS enabled devices data & $19 \mathrm{sec}$ \\
\hline 3 & Ganesh and Appavu (2015) & $\begin{array}{l}\text { Intelligent Video Systems } \\
\text { (IVS) in big data }\end{array}$ & Video data & $15 \mathrm{sec}$ \\
\hline 4 & Adoni et al. 2017 & $\begin{array}{l}\text { Traffic event detection } \\
\text { using map reduce }\end{array}$ & Apache flume & $25 \mathrm{sec}$ \\
\hline 5 & Proposed approach & Traffic event detection algorithm & Video data real time data & $10 \mathrm{sec}$ \\
\hline
\end{tabular}

\section{Conclusions and Future Work}

In this study we proposed the framework traffic event detection system for Indian traffic control system. We have proposed four different strategies as (1) congestion status on square, (2) efficient toll tax collection, (3) fine collection on road for the people who violate road traffic rules, (4) shortest pathfinder for drivers. This research work mainly focuses on the various strategies and their implementation with the help of the Map-Reduce framework for efficient traffic management in urban cities of India. The proposed system used Hadoop distributed platform and the Mapper Reducer function for the implementation. It will help in managing big data efficiently. Experiment results shows that the time required is less compare to the existing system and the proposed strategies are needed for the current state-o-the art traffic management system. In future, it be extended by adding other strategies such as adaptive traffic signal management, mobile computing platform for ease of user and traffic management authority.

\section{Acknowledgement}

The author would like to thank the Department of information Technology of, the Yeshwantrao Chavan College of Engineering, Nagpur, for providing experimental research in this study. The author would like to especially thank anonymous reviewers who took the time to make some necessary inputs.

\section{Author's Contributions}

Mr. Yogesh Golhar: Contributed to define the research problem, formulation of methods, implementation and writing the paper.

Manali Kshirsagar: Assisted in the implementation of methods and conduct of the experiment. Also Contributed on the workflow design of the manuscript.

\section{Ethics}

This article is original and contains unpublished material. Both the corresponding author and the co-author confirm that 
they have read and approved the manuscript and that no ethical issues are involved. The authors declare that they have no competing interests.

\section{References}

Dobbs, R., Smit, S., Remes, J., Manyika, J., Roxburgh, C., \& Restrepo, A. (2011). Urban world: Mapping the economic power of cities. McKinsey Global Institute, 62.

Abbu, N., Bhagavatula, L., Ghorpade, A. R., Kolsepatil, N., Kumar, E., Parvathapuram, R., ... \& Sharma, S. (2015). Urban green growth strategies for indian cities. published by Local Governments for Sustainability, South Asia, 1.

Adoni, W. Y. H., Nahhal, T., Aghezzaf, B., \& Elbyed, A. (2017, April). The MapReduce-based approach to improve vehicle controls on big traffic events. In 2017 International Colloquium on Logistics and Supply Chain Management (LOGISTIQUA) (pp. 1-6). IEEE. https://ieeexplore.ieee.org/abstract/document/7962864

Calvert, S. C., Taale, H., Snelder, M., \& Hoogendoorn, S. P. (2018). Improving traffic management through consideration of uncertainty and stochastics in traffic flow. Case Studies on Transport Policy, 6(1), 81-93. doi.org/10.1016/j.cstp.2018.01.003

Cárdenas-Benítez, N., Aquino-Santos, R., MagañaEspinoza, P., Aguilar-Velazco, J., Edwards-Block, A., \& Medina Cass, A. (2016). Traffic congestion detection system through connected vehicles and big data. Sensors, 16(5), 599. doi.org/10.3390/s16050599

De Souza, A. M., Brennand, C. A., Yokoyama, R. S., Donato, E. A., Madeira, E. R., \& Villas, L. A. (2017). Traffic management systems: A classification, review, challenges and future perspectives. International Journal of Distributed Sensor Networks, 13(4), 1550147716683612. doi.org/10.1177/1550147716683612

Ganesh, R. B., \& Appavu, S. (2015). An intelligent video Surveillance Framework with big data management for Indian road traffic system. International Journal of Computer Applications, 123(10). doi.org/10.5120/ijca2015905519

Gupta, A., Choudhary, S., \& Paul, S. (2013, September). DTC: A framework to Detect Traffic Congestion by mining versatile GPS data. In 2013 1st International Conference on Emerging Trends and Applications in Computer Science (pp. 97-103). IEEE. doi.org/10.1109/ICETACS.2013.6691403
Javaid, S., Sufian, A., Pervaiz, S., \& Tanveer, M. (2018, February). Smart traffic management system using Internet of Things. In 2018 20th international conference on advanced communication technology (ICACT) (pp. 393-398). IEEE. doi.org/10.23919/ICACT.2018.8323770

Kim, S., Kim, D. Y., \& Park, J. H. (2018). Traffic management in the mobile edge cloud to improve the quality of experience of mobile video. Computer Communications, 118, 40-49. doi.org/10.1016/j.comcom.2017.09.001

Latif, S., Afzaal, H., \& Zafar, N. A. (2018, March). Intelligent traffic monitoring and guidance system for smart city. In 2018 International Conference on Computing, Mathematics and Engineering Technologies (iCoMET) (pp. 1-6). IEEE. doi.org/10.1109/ICOMET.2018.8346327

Liu, H. Y., Skjetne, E., \& Kobernus, M. (2013). Mobile phone tracking: In support of modelling trafficrelated air pollution contribution to individual exposure and its implications for public health impact assessment. Environmental Health, 12(1), 1-12. doi.org/10.1186/1476-069X-12-93

Manikonda, P., Yerrapragada, A. K., \& Annasamudram, S. S. (2011, October). Intelligent traffic management system. In 2011 IEEE Conference on Sustainable Utilization and Development in Engineering and Technology (STUDENT) (pp. 119-122). IEEE. https://ieeexplore.ieee.org/abstract/document/6089337

Nandury, S. V., \& Begum, B. A. (2015, August). Smart WSN-based ubiquitous architecture for smart cities. In 2015 International Conference on Advances in Computing, Communications and Informatics (ICACCI) (pp. 2366-2373). IEEE.

https://ieeexplore.ieee.org/abstract/document/7275972

Swathi, K., Sivanagaraju, V., Manikanta, A. K. S., \& Kumar, S. D. (2016). Traffic density control and accident indicator using WSN. Traffic, 2(04). https://www.academia.edu/download/44713633/15.I JMTST020409.pdf

Wiering, M. A., Veenen, J. V., Vreeken, J., \& Koopman, A. (2004). Intelligent traffic light control. file:///C:/Users/PC/Downloads/wiering_04_intellige nt_traffic.pdf 